\title{
Shape-dependent magnetic properties of Co nanostructure arrays synthesized by pulsed laser melting
}

\author{
${ }^{1}$ N. Shirato, ${ }^{2}$ S. Sherrill, ${ }^{3}$ A. K. Gangopadhyay, ${ }^{1,4,5}$ R. Kalyanaraman ${ }^{1}$ \\ ${ }^{1}$ Department of Materials Science and Engineering, University of Tennessee, Knoxville, TN 37996 \\ ${ }^{2}$ Department of Materials Science and Engineering, Georgia Institute of Technology, GA 30332 \\ ${ }^{3}$ Department of Physics and Institute of Materials Science and Engineering, Washington University in St. Louis, St. \\ Louis, MO 63132 \\ ${ }^{4}$ Sustainable Energy Education and Research Center (SEERC), University of Tennessee, Knoxville, TN 37996 \\ ${ }^{5}$ Department of Chemical and Biomolecular Engineering, University of Tennessee, Knoxville, TN 37996
}

\begin{abstract}
One dimensional (1D) magnetic nanowires show unique magnetic behaviors, such as large coercivity and high remanence, in comparison to the bulk and thin film materials. Here, planar arrays of Co nanowires, nanorods and nanoparticles were fabricated from thin Co films by a nanosecond pulsed laser interference irradiation technique. Magnetic force microscopy (MFM) and surface magneto-optic Kerr effect (SMOKE) techniques were used to study the individual and average magnetic properties of the nanostructures. Magnetic domain orientation was found to depend on the in-plane aspect ratio of the nanostructure. The magnetic orientation was out-of-plane for in-plane aspect ratio ranging from 1 to 1.4 and transitioned to an in-plane orientation for aspect ratios greater than 1.4 (such as in nanorods and nanowires). Our results also showed that polycrystalline Co nanowires showed much higher coercivity and remanence as compared to bulk and thin film materials, as well as shapes with smaller aspect ratio. This result was attributed mainly to the shape anisotropy. This study demonstrated that nanosecond pulsed laser synthesis is capable of fabricating various nanostructures in a simple, robust and rapid manner and SMOKE is a reliable technique to rapidly characterize such magnetic nanostructures.
\end{abstract}

\section{Introduction}

Magnetic properties show important changes from bulk behavior when the sample dimension is reduced to length scales comparable with the magnetic domain wall width or exchange length. Most common examples are multiple- to single-domain behavior and changes in switching modes with the external magnetic field. In addition to the size, other factors such as shape, crystalline state (single vs. polycrystal), and strain, become increasingly important for their magnetic properties. Both scientific curiosity and potential applications continue to drive interest in this field. The choice of materials in a variety of applications, such as perpendicular or longitudinal high density magnetic storage [1], non-volatile and high speed magnetic memories (MRAM) [2, 3], opto-electronics [4] and biological applications [5, 6, 7, 8] require magnetization to be in a preferred direction (in-plane or out-of-plane). In contrast to bulk materials, where intrinsic properties such as crystalline anisotropy controls the magnetization direction, extrinsic effects (shape anisotropy, strain) play much more important roles in determining the magnetization direction of nanomaterials. To

\footnotetext{
${ }^{1}$ All correspondence to be addressed to ramki@utk.edu
} 
exploit these extrinsic effects, a significant amount of research has been devoted to magnetic nanowires and magnetic nanoparticles $[9,10]$. For instance, a magnetic nanowire with width comparable to the domain wall shows large coercivity, large anisotropy and high remanence due to the shape and the magnetocrystalline anisotropy [11].

Another approach towards controlling the magnetic behavior is to utilize non-equilibrium processing techniques that can result in quenched in crystal structures and/or novel shapes. One general approach is to utilize pulsed energetic beams which can deliver large amounts of energy in short durations, such as ion or pulsed laser beams. As shown recently, nanosecond pulsed laser liquid state processing $[12,13]$ produces different magnetic behavior within nanoparticles as compared to solid state thermal annealing [14]. Specifically, synthesis of hemispherical ferromagnetic metal nanoparticles by pulsed laser dewetting produce single domain nanoparticles with preferred orientations due to a combination of their granular nature and residual thermal strain from the fast laser processing [13, 12]. However, to the best of our knowledge, study of the ensuing magnetic behavior in the same metal/alloy system as the nanostructure evolves continuously from long nanowires to isolated nanoparticles has not been reported. Such a study would help to quantify the changing magnetic behavior with shape, especially the in-plane aspect ratio AR, defined here as the ratio of the length to width of the nanowires measured along the substrate plane. We present here results from such an investigation on Co nanostructures using the surface magneto-optic Kerr effect (SMOKE) [15] and magnetic force microscopy (MFM) techniques. The former was used for average magnetic properties and the latter for the individual nanostructures. Arrays of Co nanostructures of various in-plane aspect ratios were prepared by applying repeated high-intensity pulsed nanosecond laser interference patterns on ultra-thin films. The molten films evolved continuously into long nanowires (nanostructures with lengths orders of magnitude larger than other dimensions), followed by nanorods ( lengths larger then the width), and finally into hemispherical nanoparticles with increasing number of pulses [16].

The magnetic remanence, coercivity, and domain behavior studied by SMOKE and MFM techniques of the various Co nanostructures (nanowires, nanorods and nanoparticles) suggest that the aspect ratio is the most important factor that controls their magnetic properties.

\section{Experimental Details}

\section{Synthesis of 1-D nanowire arrays by two beam interference irradiation}

Co thin films of $6 \mathrm{~nm}$ thickness were deposited onto commercially available and optically smooth 400 $\mathrm{nm}$ thermally grown amorphous $\mathrm{SiO}_{2}$ on $\mathrm{Si}(100)$ wafers by using the electron-beam evaporation technique under ultra-high vacuum (base pressure of $1 \times 10^{-8}$ Torr) conditions. We have shown previously that such substrates provide a good combination of the low surface energy required for dewetting and transport of the metal film following laser melting and do not show evidence for chemical reactions with the metallic films $[16,17]$. The substrates were ultrasonically cleaned with acetone and isopropanol before deposition. The film was deposited through a periodic array of square nylon contact masks with $280 \mu \mathrm{m}$ by $280 \mu \mathrm{m}$ openings, spaced at a distance of $400 \mu \mathrm{m}$. This produced a patterned surface comprising of a large number of film islands that could be independently irradiated and investigated, thereby increasing the efficiency of the experimental investigations. The film thickness was calibrated by step-height measurements with an atomic force microscope (AFM). Following the deposition, two beam laser interference irradiation was performed under ambient conditions using a $266 \mathrm{~nm}$ ultraviolet (UV) laser with a $9 \mathrm{~ns}$ pulse duration of sufficient energy density to melt the Co film. A $200 \mu \mathrm{m}$ diameter laser beam with Gaussian spatial profile and 100 $\mathrm{mJ} / \mathrm{cm}^{2}$ energy density was split into two equal energy density beams using a 50-50 beam splitter. They were incident perpendicular to the substrate plane at an inclusive angle of $45.7^{\circ}$ to produce an interferencepattern with $343 \mathrm{~nm}$ spacing. As shown in previous studies [18, 16], the film starts rupturing with spatially periodic structures at length scales comparable to the interference spacing with increasing number of laser pulses, $n$. The temperature transients from the ns laser pulse result in heating and subsequent cooling rates that 
approach $\sim 10^{10} \mathrm{~K} / \mathrm{s}$. The primary mass transport occurs during the molten phase, which typically lasts between 10-50 ns for each pulse. Therefore, the nanostructure formation under laser interference can be summarized as a result of mass transport via thermal gradient and hydrodynamic forces and subsequent rapid quenching-in of the existing structure. In this framework, smaller number of pulses lead to the formation of nanowires since thermal gradient effects dominate the early stages of mass transport, while a larger number of pulses lead to the break-up of the nanowires via the Rayleigh instability, yielding smaller sized features $[17,16]$. In the early stages of transformation of the film, nanostructures consisting of two thin parallel wires connected by a groove formed, which are referred to here as "grooved wire" (GW) [Fig. 1(a)]; later, they transformed into isolated "single-wires" [SW, see Fig. 1(b)], nanorods [Fig. 1(c)] and finally, into nanoparticles [NP, Fig. 1(d)]. Each image corresponded to a different Co film sample, but of identical thickness, exposed to a different number of pulses. Typically, 10 pulses were required for the formation of GW, 100 pulses for the nanowires, 500 pulses for the nanorods, and 1000 pulses for the nanoparticles. When irradiating the metal film with a two-beam interference pattern, the resulting sinusoidally varying and periodic laser intensity induces a transient and periodic thermal gradient along the plane of the film which creates a surface tension gradient. Consequently, thermocapillary (Marangoni) convection results in the formation of long, cylindrical-like, nanowires. Further irradiation causes the Rayleigh instability of certain wavelengths, and the nanowires break up into nanorods and nanoparticles [16].

The various nanostructure morphologies were characterized by scanning electron microscopy (SEM, a Hitachi S4300N and a Zeiss Merlin) and atomic force microscopy (AFM, Asylum MFP-3D). The magnetic domain orientation of the nanostructures with respect to the substrate was determined from the bright and dark contrast of the magnetic force microscopy (MFM). The details of the MFM measurement have been published elsewhere [13]. The magnetic switching behavior of the nanostructure arrays were characterized by surface magneto-optic Kerr effect (SMOKE) technique [15] using a set-up detailed in ref. [19]. In this system, a monochromatic $635 \mathrm{~nm}$ diode laser beam ( $80 \mu \mathrm{m}$ spot size) was polarized by passing through a Glan-Thompson polarizer and then focussed onto the sample. This laser spot size was well suited to probe regions containing the pulsed laser irriadiated zones containing the variousnanostructures of interest, such as nanowires or nanoparticles. SMOKE measurements were performed on the samples in two different geometries, the polar geometry in which the magnetic field was perpendicular to the substrate plane, and longitudinal geometry in which the magnetic field was parallel to the substrate plane. For the longitudinal geometry, the external magnetic field was applied either parallel or perpendicular to the wire direction in order to determine preferential magnetization direction. The beam was incident normal to the substrate plane at angles of $\theta_{i}=72.4^{\circ}$ for the polar and $\theta_{i}=17.6^{\circ}$ for the and longitudinal measurements.

\section{Results}

\subsection{SEM, AFM and MFM}

From the SEM images of the various structures, histograms of the width and length were generated from three randomly selected pattered areas of $\sim 2 \mu \mathrm{m}^{2}$. In Figs. 2(a) and (b), the various histograms represent the width and length distributions of single-wires, nanorods and nanoparticles. From Fig. 2(a), the average widths of the single-wires and nanorods were similar [within 9\%], whereas the average width (= diameter) of nanoparticles was $29 \%$ larger than that of the nanowires and nanorods. From Fig. 2(b), the average length of the single-wire was $78 \%$ and $92 \%$ larger than the nanorod and nanoparticle, respectively. The arrays of both nanowires had an average spacing of $377 \pm 27 \mathrm{~nm}$. The arrays of the single-wire and groovedwire had $92 \pm 6 \mathrm{~nm}$ and $35 \pm 5 \mathrm{~nm}$ (width of thin elongated part but not width of the groove) average width, respectively. From Fig. 1(d), the average spacing of ordered nanostructures perpendicular to the nanowire direction was measured to be $369 \pm 23 \mathrm{~nm}$. Also, the average length and width of nanorods was estimated to be $351 \mathrm{~nm}$ and $82 \pm 8 \mathrm{~nm}$ [1(d)], while the average diameter of the particles was estimated to be $132 \pm 24 \mathrm{~nm}$ [Fig. 1(e)]. 
The AFM height profile of Co nanostructures are shown in the left side images of Figs. 3(a) - (d), with the corresponding MFM images on the right side. The AFM and MFM images were obtained simultaneously from the sample through topography and phase imaging. From the AFM image contrast in Fig. 3(a) from the grooved wires, the groove-like morphology at the center of the each wire can be observed; however due to the comparable size of the AFM tip $(\mathrm{R}=50 \mathrm{~nm})$ to the groove width $(50 \mathrm{~nm})$ and depth $(60 \mathrm{~nm})$, the AFM image could not resolve the groove height profile clearly. The MFM images showed multiple magnetic domains in the single-wires, oriented parallel to the wire lengths (alternative bright and dark regions along the wires). The single nanowires are shown in Fig. 3(b), and the MFM contrast again showed domains oriented parallel to the wire length. The nanorod/nanoparticle image in Fig. 3(c) showed some important changes as the elongated particles still showed a domain orientation along the original nanowire length while the particles appeared to have an out of plane orientation. This was clearly evident in the nanoparticle sample, Fig. 3(d), which showed single domain behavior with the particles preferentially showing an out-of-plane magnetic orientation that was independent of the spatially ordered direction.

\subsection{Magnetization measurements by SMOKE}

The primary goal of our SMOKE measurements was to confirm the average magnetic properties of each of the three types of nanostructure arrays (nanowires, nanorods and nanoparticles). From SMOKE, the Kerr signal vs magnetic field $\mathrm{H}$ hysteresis loop can be generated, which in turn provides the average saturation field $\mathrm{H}_{S}$ and coercivity $\mathrm{H}_{C}$ of the arrays over the region probed by the optical beam. When this measurement is done in the polar and longitudinal geometries, it is possible to differentiate between ease of domain rotation along in-plane and out-of-plane orientations. For example, when the array has smaller magnetic saturation field in polar geometry versus longitudinal, it would indicate easier out-of-plane magnetization axis, such as observed by us previously in hemispherically shaped nanoparticles prepared by the laser melting and dewetting technique $[12,13]$. Similarly, a smaller saturation field in the longitudinal geometry would indicate preferred in-plane magnetization. In fact, when SMOKE measurements are done as a function of angle, the exact orientation of the magnetic axis could, in principle, be determined.

Fig. 4(a) shows the Kerr signal generated hysteresis loops of Co grooved wire [Fig. 1(a)] array for two different SMOKE geometries. The red lines represent data for the magnetic field applied in-plane, parallel to the wire direction (longitudinal geometry, $L^{\|}$) and the blue lines for the perpendicular direction ( longitudinal geometry, $L^{\perp}$ ). Coercivity values in the $L^{\|}$and $L^{\perp}$ geometries were $H_{c}^{\|}=1000 \mathrm{Oe}$ and $H_{c}^{\perp}=432 \mathrm{Oe}$, and the saturation fields were $H_{s}^{\|}=2.1$ and $H_{s}^{\perp}>3 \mathrm{kOe}$, respectively. The larger coercivity and smaller saturation fields for $L^{\|}$compared to $L^{\perp}$ indicated that the magnetization of Co grooved wire arrays were preferentially oriented along the in-plane wire axis. This was consistent with the MFM images of individual nanowires [Fig. 1(a, b)] and with previous studies [20, 21, 22].

Fig. 4(b) shows hysteresis loops of ordered Co nanoparticle arrays for the two longitudinal geometries, $L^{\|}$and $L^{\perp}$. The inset plot compares the hysteresis loop for the polar geometry with the $L^{\|}$and $L^{\perp}$ orientations. Coercivity values in the $L^{\|}$and $L^{\perp}$ were negligible, and saturation fields were $H_{s}^{\|}=4.2 \mathrm{kOe}$ and $H_{s}^{\perp}=$ $4.1 \mathrm{kOe}$, respectively. Almost identical hysteresis loops in the $L^{\|}$and $L^{\perp}$ geometries suggested that the nanoparticle array no longer has the shape anisotropy along the sample plane due to the hemispherical shapes. Moreover, much higher saturation fields than the grooved wires in both in-plane field indicated that the easy-axis was not in the substrate plane. Although the hysteresis loop in the polar geometry (inset) also looked similar (small coercivity), it was different from the longitudinal geometry in one important aspect; the Kerr signals were much stronger in the polar geometry compared to the longitudinal ones. These results indicate that the average magnetization direction of the nanoparticles are out-of-plane, probably at some angle with the substrate plane, which is consistent with the MFM results.

Fig. 5(a) shows hysteresis loops of each nanostructure array including the grooved-wire, single-wire and rods/particles in the $L^{\|}$geometry. The hysteresis loops indicated that the coercivity decreased when going 
from wires to rods, or as the aspect ratio decreased. The grooved wire $\left(H_{c}^{\|}=1000 \mathrm{Oe}\right)$, which has a different shape and geometry compared to the single-wire, had higher coercivity than the single-wire $\left(H_{c}^{\|}=670 \mathrm{Oe}\right)$. Also, the single-wire, which has a larger aspect ratio than the rod, had higher coercivity than the rod $\left(H_{c}^{\|}\right.$ $=340 \mathrm{Oe}$ ) . Fig. 5(b) compares the hysteresis loops of the grooved wire, rods/particles and nanoparticle arrays in the polar geometry. It indicated that for any applied field, the Kerr signal from the particles was stronger than the nanowires. However, for these polar measurements, magnetic fields much stronger than 6 kOe (beyond that available to us during these measurements) were required for saturation and therefore, it was not possible to determine the saturation fields for the three different nanostructures probed in Fig. 5(b).

\section{Discussion}

From the AFM and MFM images, the role of the AR of a nanostructure in relation to its corresponding magnetic domain type and magnetization orientation could be compared. In Fig. 6, the histogram represents the AR distribution of nanostructures with its magnetic domain and magnetization orientation. First, the histogram indicated that the single domain nanostructures exists in the range of $1<A R<3$ while multi domain was found for $A R \geq 3$. Second, the histogram showed the locations of out-of-plane and in-plane magnetizations. Single domain and out-of-plane magnetization was observed for $1<A R<1.4$, single domain with in-plane magnetization was observed for $1.4 \leq A R<3$, while multi-domain in-plane magnetization was found for $A R \geq 3$. Earlier studies on Co nanoparticles prepared by similar laser melting and dewetting technique on similar substrates $\left(\mathrm{SiO}_{2}\right.$ on $\mathrm{Si}(100)$ wafers) showed $[13,12]$ that the residual tensile thermalmismatch strain from the substrate and the negative magnetostriction coefficient of Co causes the magnetic moment to orient out-of-plane (at some finite angle to the substrate). Since such strains are similar for all Co-nanostructures studied here, the difference in magnetic orientations of the nanostructures must then be a result of shape anisotropy. For structures with large aspect ratio $(A R>>1$, i.e. nanowires, nanorods), the shape anisotropy wins over strain-induced anisotropy (the magnetocrystalline anisotropy is averaged out because of random orientation of crystal grains in these polycrystals) and the magnetic moments align along the long-axis. At smaller AR, strain-induced anisotropy wins and the magnetic moments of the single particles align out-of-substrate-plane. There is a transition region for $A R \sim 1.4$, as shown by the thin-dotted vertical line in Fig. 6.

To understand the relation between observed coercivity values and aspect ratio of the nanostructures, we employed the chain of spheres model developed by Jacobs and Bean [23, 24]. We assumed here that each nanowire and rod is made from continuous single domain spheres, the chain of spheres, where only a point contact occurs between the spheres and hence, the exchange force could be treated as being discontinuous at the boundary. Therefore, magnetization reversal was assumed to occur independently for each particle in the chain of spheres $[23,21]$. The coercivity can then be obtained from the following expressions [23]:

$$
H_{n}=\left(\frac{\mu}{a^{3}}\right)\left(6 K_{n}-4 L_{n}\right)
$$

where $\mu$ is the dipole moment of a sphere of diameter $a$, and $n$ is the number of spheres in the nanowire or rod. The dipole moment was defined as:

$$
\mu=M_{s}\left(\frac{4 \pi}{3}\right)\left(\frac{a}{2}\right)^{3}
$$

where, $M_{s}$ is the saturation magnetization of Co. The remaining quantities in eq. 1 were given as:

$$
L_{n}=\sum_{j=1}^{\frac{1}{2}(n-1) \leq j \leqq \frac{1}{2}(n+1)} \frac{[n-(2 j-1)]}{n(2 j-1)^{3}} ; \text { and } K=\sum_{j=1}^{n j} \frac{(n-j)}{n j^{3}}
$$


where $i$ and $j$ are integers ranging from $l$ to $n$.

From the histograms in Fig. 2( and b), the single-wire (top panels), nanorod (middle panels), and nanoparticle (bottom panels) had average aspect ratio of $\sim 17.4,4.4$ and 1.1, respectively, with aspect ratio $=$ length / width. For the above calculation, the number of spheres, $n$, was taken as equivalent to the aspect ratio. This was justified since both the nanowire and nanorods had comparable width ( $6 \%$ difference) and hence $n \approx$ aspect ratio. In Fig. 7, the experimentally measured coercivity values of nanostructures with respect to the aspect ratio are plotted along with the values calculated from the model. The solid line represents the best fit line of the experimental data points with respect to the aspect ratio based on the model. The trend of the experimental data points and the model calculation were consistent with each other, i.e. both showed an increasing coercivity with increasing aspect ratio. In addition, the intrinsic coercivity value, $H_{c i}$ $\left(=\mu / a^{3}\right)$, could be calculated from the fitted data and yielding $H_{c i, f i t}=250(\mathrm{Oe})$, which was three times smaller than the previously reported value $[25,23]$. One explanation for this could be the difference in width of our structures compared with the previous study. The average width of our samples were approximately $85 \%$ smaller then the width of nanowires of the previous study, and could be the reason for this mismatch [21]. Alternately, it should be emphasized that magnetization reversal can also occur by a coherent rotation [23], as compared to the incoherent case used here to describe the macroscopic magnetization reversal. In the future, we plan to extend this analysis ti include the predictions from coherent rotation.

\section{Conclusion}

In conclusion, we successfully fabricated various nanostructures of ferromagnetic Co in the form of nanowires, nanorods and nanoparticles using the nanosecond pulsed laser synthesis technique. The magnetic switching behavior and domain property of the arrays were studied using SMOKE and MFM characterization techniques. We found that magnetic domain properties depended on the ratio of length to width, i.e. the aspect ratio. The magnetization direction was out-of-plane for nanostructures with aspect ratio, $1<A R<1.4$ and transitioned to an in-plane orientation for aspect ratios greater than 1.4. From the hysteresis loop measurements of Co nanostructure arrays using SMOKE, large in-plane magnetic anisotropy was observed for Co nanowires along the wire direction. On the other hand, no large in-plane magnetic anisotropy was observed for Co nanoparticles. This result was mainly attributed to the shape anisotropy. The relation between the nanostructure coercivity and the aspect ratio was also evidenced by the chain of spheres model.

\section{Acknowledgments}

The authors acknowledge support by NSF DMR-0856707, the Sustainable Energy Education and Research Center, while RK also acknowledges support through ARO grant W911NF-13-1-0428. A portion of this research was conducted under grant CNMS2011-R78 at the Center for Nanophase Materials Science, which is sponsored at ORNL by the Scientific User Facilities Division, Office of Basic Energy Sciences, U.S. Department of Energy. One of the co-authors, Sidney Sherril, was an undergraduate student supported by an NSF REU summer research grant.

[1] P. R. Krauss, P. B. Fischer, S. Y. Chou, Fabrication of single-domain magnetic pillar array of $35 \mathrm{~nm}$ and 65 bits/in² density, J. Vac. Sci. Technol. B 12 (1994) 3639.

[2] R. M. H. New, R. F. W. Pease, R. L. White, J. Vac Sci. Technol. B 12 (1994) 3196.

[3] S. Y. Chou, P. R. Krauss, L. Kong, Nanolithographically defined magnetic structures and quantum magnetic disk, J. Appl. Phys. 79 (1996) 6101. 
[4] M. Salerno, J. R. Krenn, B. Lamprecht, G. Schider, H. Ditlbacher, N. Felidj, A. Leitner, F. R. Aussenegg, Opto-Electron. Rev. 10 (2002) 217.

[5] R. S. Molday, D. Mackenzie, J. Immunol. Methods 52 (1982) 353.

[6] A. Jordan, R. Scholz, P. Wust, H. Schirra, T. Schiestel, H. Schmidt, R. Felix, J. Magn. Magn. Mater. 194 (1999) 185.

[7] D. H. Reich, M. Tanase, A. Hultgren, L. A. Bauer, C. S. Chen, G. J. Meyer, Biological applications of multifunctional magnetic nanowires (invited), Journal of Applied Physics 93 (10) (2003) 7275-7280. URL http://link.aip.org/link/?JAP/93/7275/1

[8] E. J. Felton, D. H. Reich, Biological Applications of Multifunctional Magnetic Nanowires, John Wiley \& Sons, Inc., 2007, pp. 1-22. doi:10.1002/9780470152928.ch1.

URL http://dx.doi .org/10.1002/9780470152928.ch1

[9] D. Sellmyer, M. Zheng, R. Skomski, Magnetism of fe, co and ni nanowires in self-assembled arrays, J. Phys.: Condens. Matter 13 (2001) R433-R460.

[10] R. Skomski, Nanomagnetics, J. Phys.: Condens. Matter 15 (2003) R841-R896.

[11] H. Zeng, R. Skomski, L. Menon, Y. Liu, S. Bandyopadhyay, D. J. Sellmyer, Structure and magnetic properties of ferromagnetic nanowires in self-assembled arrays, Physical Review B 65 (13) (2002) 134426-. doi:10.1103/PhysRevB.65.134426.

URL http://link.aps.org/doi/10.1103/PhysRevB.65.134426

[12] H. Krishna, A. K. Gangopadhyay, J. Strader, R. Kalyanaraman, Nanosecond laser-induced synthesis of nanoparticles with tailorable magneticanisotropy, Journal of Magnetism and Magnetic Materials 323 (3-4) (2011) 356-362.

URL http://www.sciencedirect.com/science/article/pii/S0304885310006840

[13] H. Krishna, C. Miller, L. Longstreth-Spoor, Z. Nussinov, A. K. Gangopadhyay, R. Kalyanaraman, Unusual size-dependent magnetization in near hemispherical co nanomagnets on sio[sub 2] from fast pulsed laser processing, Journal of Applied Physics 103 (7) (2008) 073902. doi:10.1063/1.2901198.

URL http://link.aip.org/link/?JAP/103/073902/1

[14] Y.-J. Oh, J.-H. Kim, C. V. Thompson, C. A. Ross, Templated assembly of co-pt nanoparticles via thermal and laser-induced dewetting of bilayer metal films, Nanoscale 5 (2013) 401-407. doi:10.1039/C2NR32932H.

URL http://dx.doi.org/10.1039/C2NR32932H

[15] Z. Q. Qiu, S. D. Bader, Surface magneto-optic kerr effect, Review of Scientific Instruments 71 (3) (2000) 1243-1255. doi:10.1063/1.1150496.

URL http://link. aip.org/link/?RSI/71/1243/1

[16] C. Favazza, J. Trice, R. Kalyanaraman, R. Sureshkumar, Self-organized metal nanostructures through laser-interference driven thermocapillary convection, Applied Physics Letters 91 (4) (2007) 043105.

[17] C. Favazza, J. Trice, H. Krishna, R. Kalyanaraman, R. Sureshkumar, Laser-induced short-and longrange orderings of co nanoparticles on sio 2, Applied Physics Letters 88 (15) (2006) 153118-153118. 
[18] N. Shirato, J. Strader, A. Kumar, A. Vincent, P. Zhang, A. Karakoti, P. Nacchimuthu, H. J. Cho, S. Seal, R. Kalyanaraman, Thickness dependent self limiting 1-d tin oxide nanowire arrays by nanosecond pulsed laser irradiation, Nanoscale 3 (3) (2011) -.

URL http://dx.doi.org/10.1039/CONR00689K

[19] N. Shirato, H. Krishna, A. K. Gangopadhyay, R. Kalyanaraman, Magnetic measurement of pulsed laser-induced nanomagnetic arrays using surface magneto-optic kerr effect, Vol. 7767, SPIE, 2010, p. 77670Q. doi:10.1117/12.861004.

URL http://link. aip.org/link/?PSI/7767/77670Q/1

[20] Y. Henry, K. Ounadjela, L. Piraux, S. Dubois, J. M. George, J. L. Duvail, Magnetic anisotropy and domain patterns in electrodeposited cobalt nanowires, The European Physical Journal B - Condensed Matter and Complex Systems 20 (1) (2001-03-19) 35-54. doi:10.1007/s100510170283.

URL http://dx.doi.org/10.1007/s100510170283

[21] Y. Peng, H.-L. Zhang, S.-L. Pan, H.-L. Li, Magnetic properties and magnetization reversal of alpha-fe nanowires deposited in alumina film, Journal of Applied Physics 87 (10) (2000) 7405-7408.

URL http://link. aip.org/link/?JAP/87/7405/1

[22] K. Nielsch, R. B. Wehrspohn, J. Barthel, J. Kirschner, U. Gosele, S. F. Fischer, H. Kronmuller, Hexagonally ordered $100 \mathrm{~nm}$ period nickel nanowire arrays, Applied Physics Letters 79 (9) (2001) 1360-1362. URL http://link. aip.org/link/?APL/79/1360/1

[23] I. S. Jacobs, C. P. Bean, An approach to elongated fine-particle magnets, Physical Review 100 (4) (1955) 1060-1067. doi:10.1103/PhysRev.100.1060.

URL http://link.aps.org/doi/10.1103/PhysRev.100.1060

[24] B. D. Cullity, C. D. Graham, Introduction to Magnetic Materials, 2nd Edition, Wiley, 2009.

[25] C. Kittel, Theory of the structure of ferromagnetic domains in films and small particles, Physical Review 70 (11-12) (1946) 965-971. doi:10.1103/PhysRev.70.965.

URL http://link. aps .org/doi/10.1103/PhysRev.70.965 


\section{Figure Captions}

- Fig:1: SEM images of Co arrays of (a) grooved wire, (b) single wire, (c) rods/particles and (d) nanoparticles, respectively. (Co metal and $\mathrm{SiO}_{2}$ substrate regions correspond to the bright and dark contrasts respectively). The as-deposited film prior to laser irradiation showed a uniform and featureless morphology and is not shown here.

- Fig:2: The nanostructure size distribution of (a) width (W) and (b) length (L) of Co single wire, nanorod and nanoparticle arrays. The average width and length of wires and rods, and diameter of particles estimated from the histograms are $\mathrm{W}=92, \mathrm{~L}=1600 \mathrm{~nm}$ (single-wire), $\mathrm{W}=82 \mathrm{~nm}, \mathrm{~L}=357$ $\mathrm{nm}$ (rods) and $\mathrm{D}=132 \mathrm{~nm}$ (particles), respectively.

- Fig:3: AFM topography (left) and simultaneous MFM phase contrast (right) images from Co (a) grooved wire, (b) single wire, (c) rods/particles and (d) nanoparticles, respectively.

- Fig:4: Magnetic hysteresis behavior in the longitudinal geometry. (a) Comparison between the hysteresis loops for the nanowire arrays in the $\mathrm{L}^{\|}$and $\mathrm{L}^{\perp}$ geometries. (b) Comparison between the hysteresis loops for the particle arrays in the $\mathrm{L}^{\|}$and $\mathrm{L}^{\perp}$ geometries. Inset of (b) also includes data for the polar geometry.

- Fig:5: Magnetic hysteresis comparison. (a) Hysteresis from grooved wire (red curve), single wire (green curve) and rods/particles (blue curve), compared in the $\mathrm{L}^{\|}$geometry. (b) Hysteresis from grooved wire (red curve), rods/particles (purple curve) and nanoparticles (orange curve), compared in the polar geometry.

- Fig:6: Histogram of the aspect ratio (AR) distribution of nanostructures along with the nature of magnetic domain (single- vs multi-domain) and their magnetization orientation. The histogram indicates that the single domain and multi-domain nanostructure exists in the range of $1<A R<3$ and $A R \geq 3$, respectively. The AR values corresponding to out-of-plane and in-plane magnetization, as well as multi-domain magnetization are also shown. The vertical dashed line at $A R=1.4$ corresponds to the transition from out-of-plane to in-plane (for AR > 1.4) magnetization.

- Fig:7: Measured values of the in-plane coercivity of Co nanowire, nanorod, and nanoparticle arrays (open circles) compared to the theoretical trend in coercivity obtained by the chain-of-spheres model [23]. 


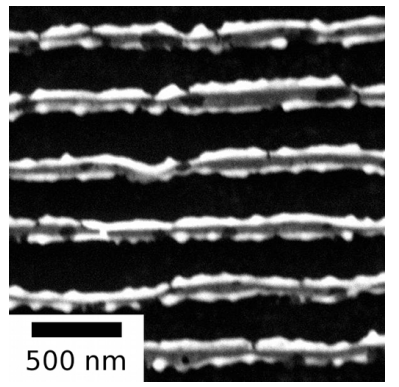

(a)

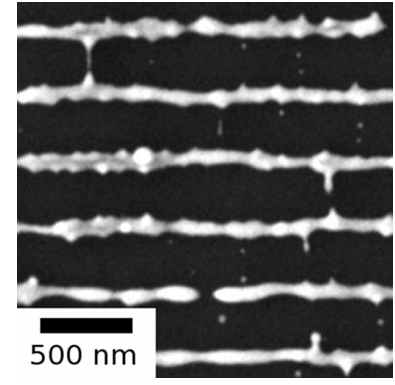

(b)

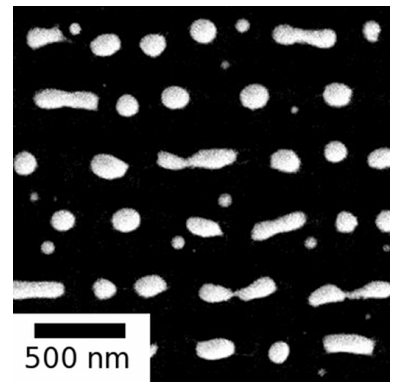

(c)

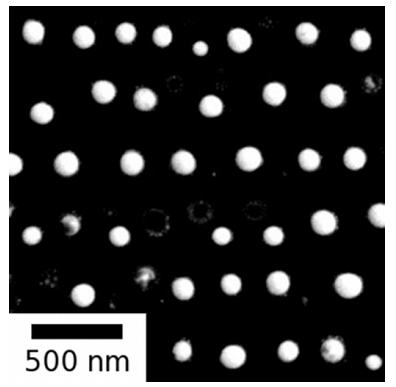

(d)

Figure 1
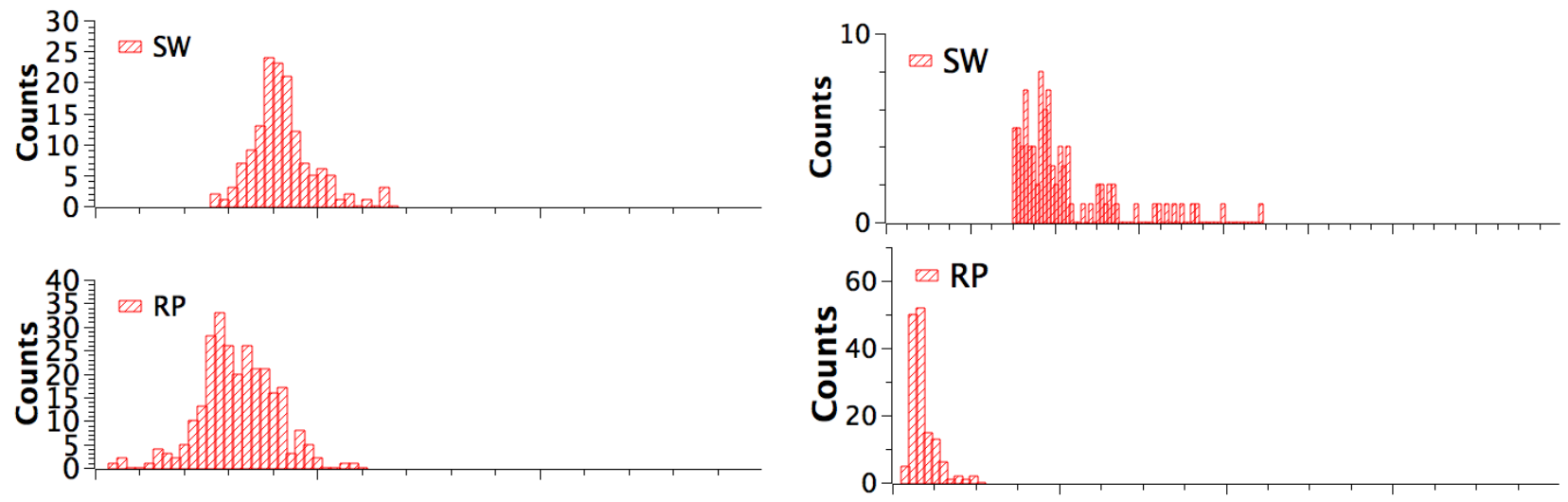

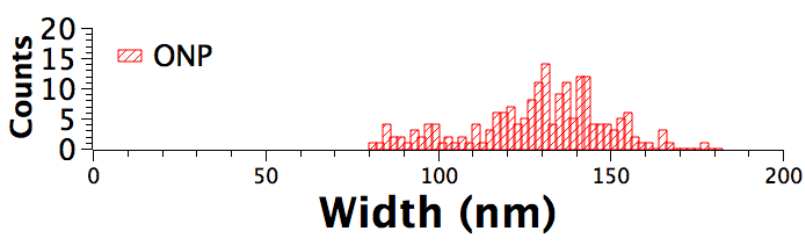

(a)

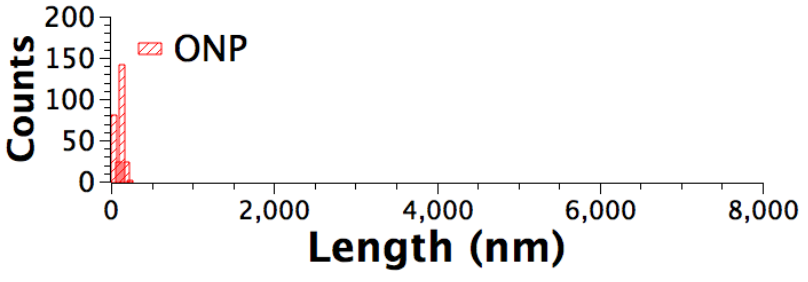

(b)

Figure 2

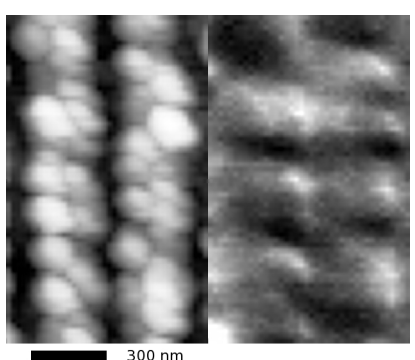

(a)

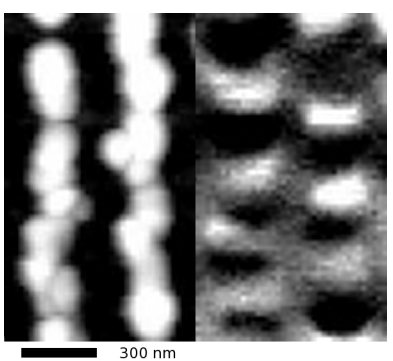

(b)

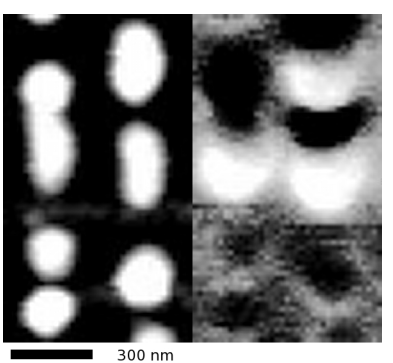

(c)

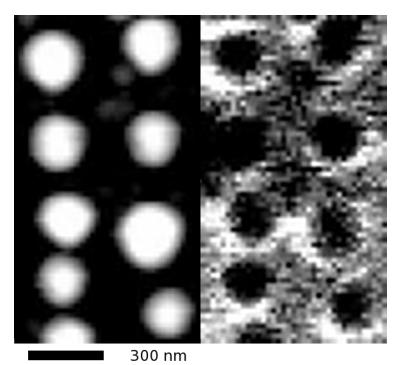

(d)

Figure 3 


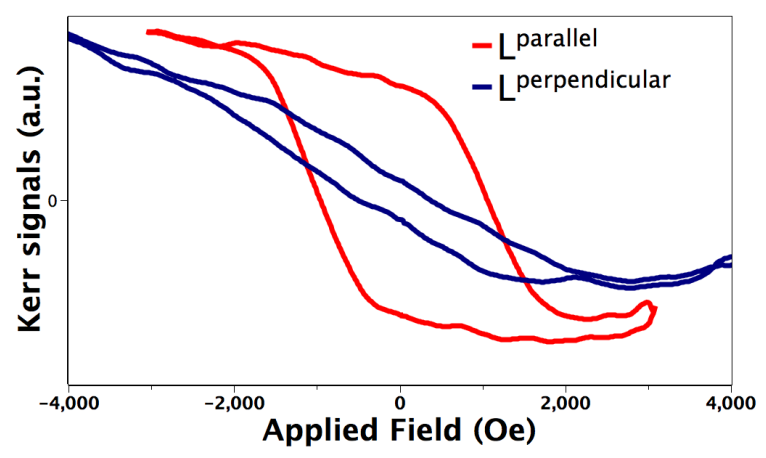

(a)

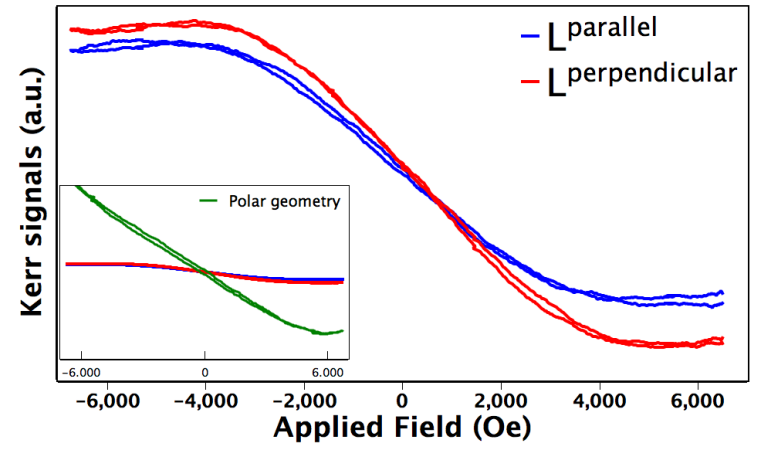

(b)

Figure 4

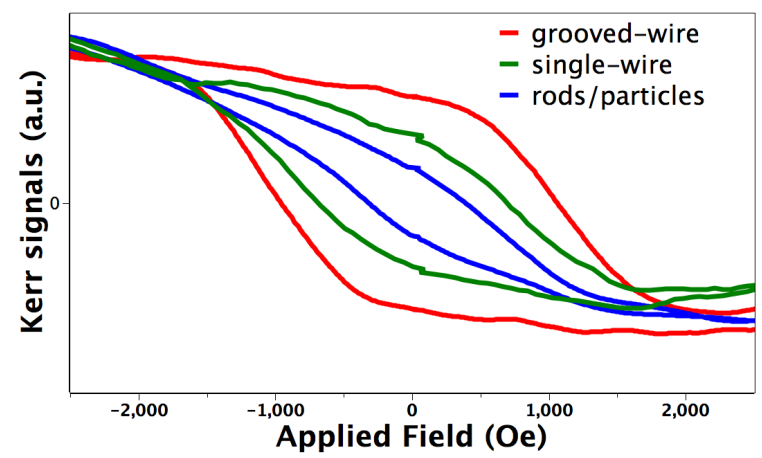

(a)

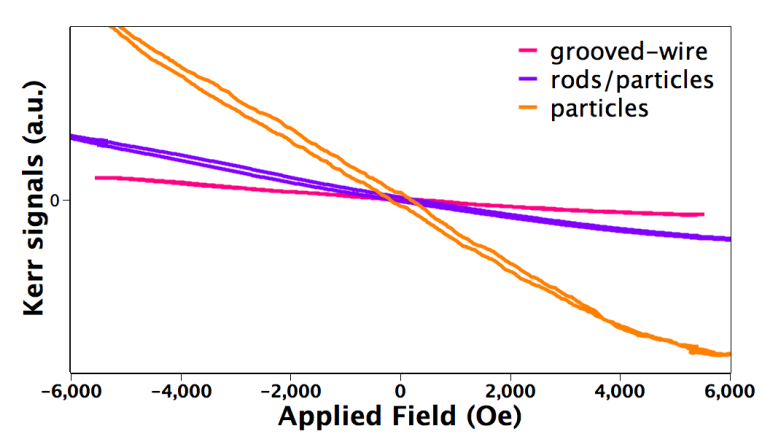

(b)

Figure 5 


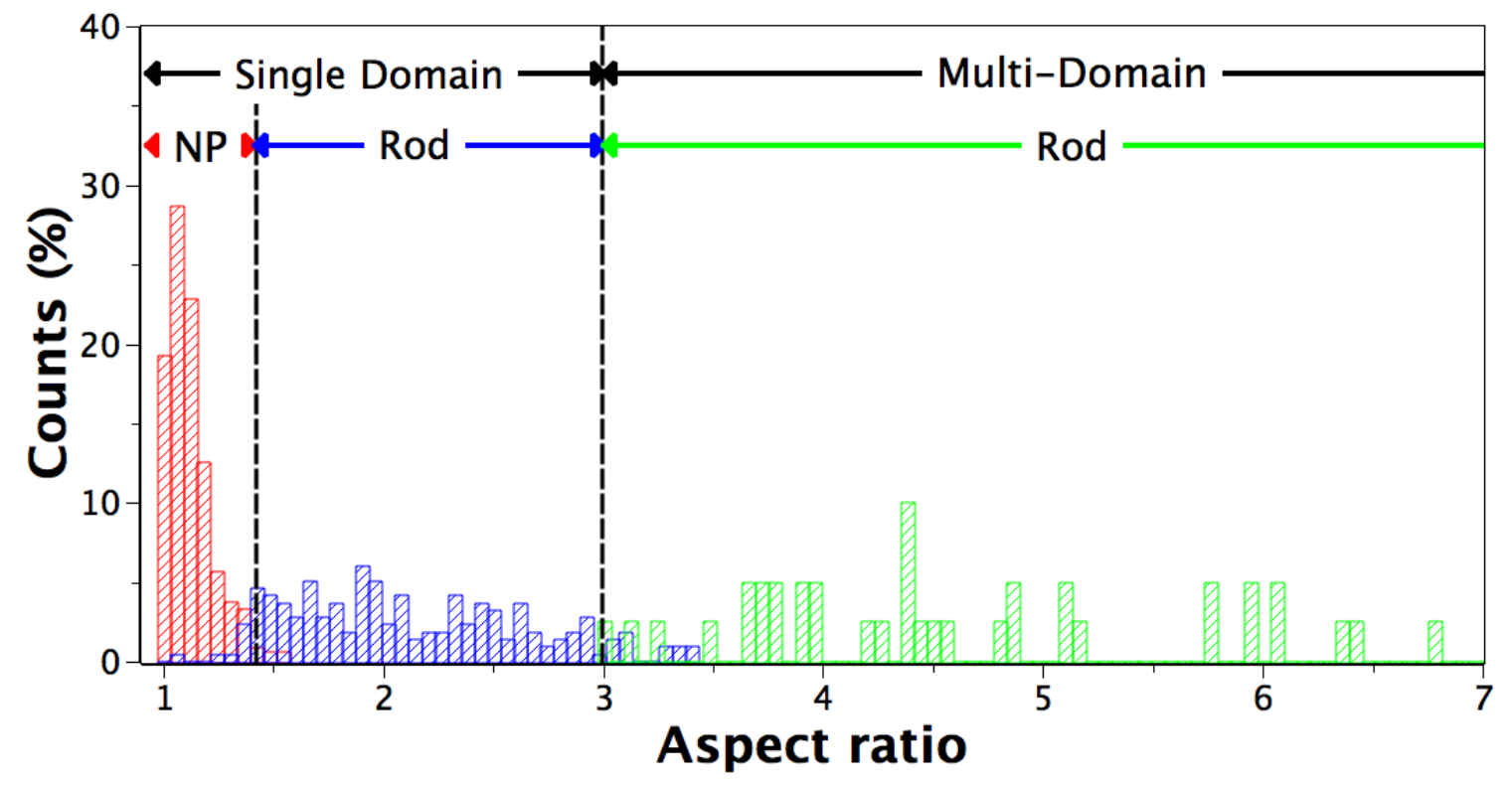

Figure 6

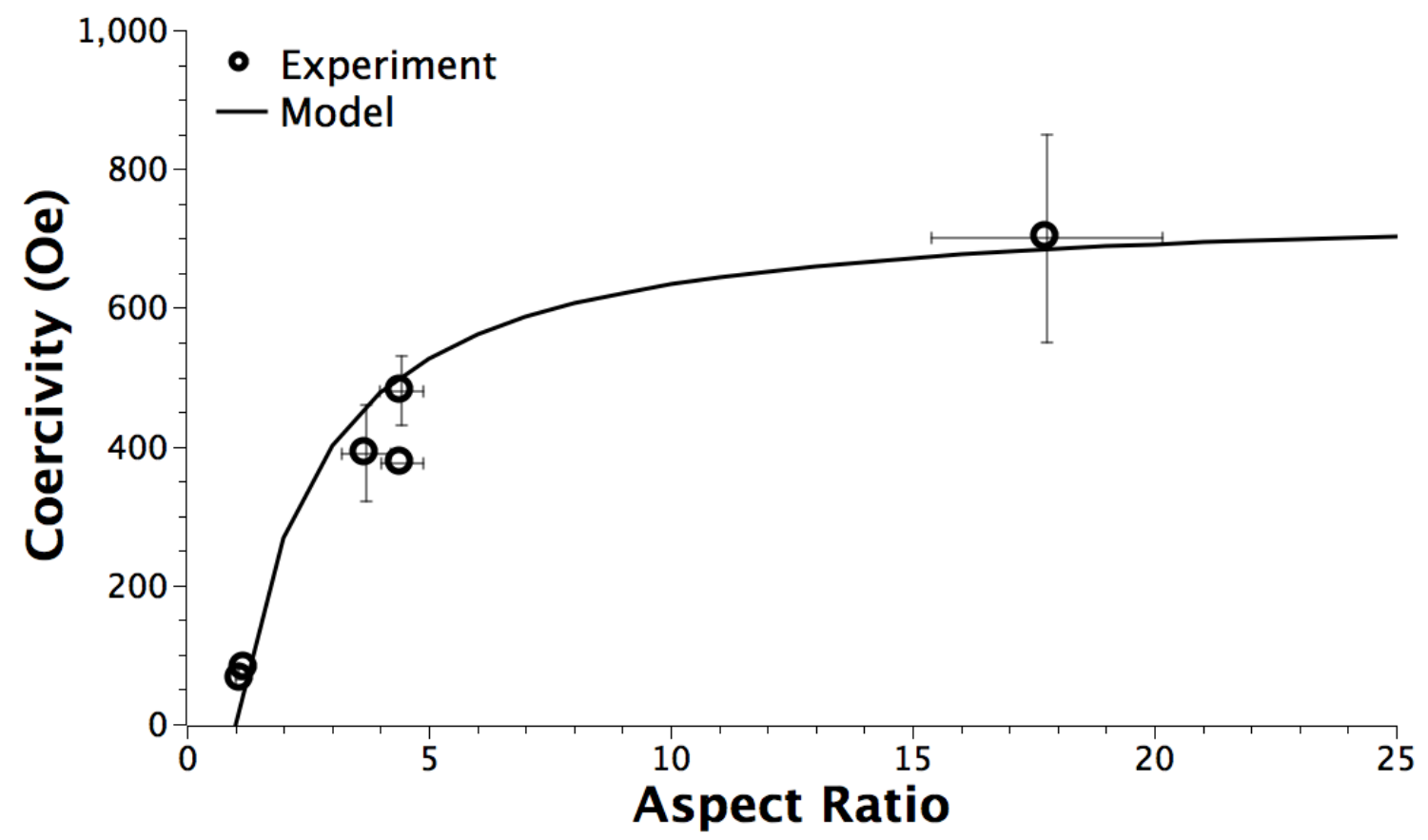

Figure 7: Measured values of the in-plane coercivity of Co nanowire, nanorod, and nanoparticle arrays (open circles) compared to the theoretical trend in coercivity obtained by the chain-of-spheres model [23]. 